Ann. Zootech., I972, 21 (I), I2I-I28.

NOTE

\title{
INFLUENCE DE L'APPORT D'ALIMENT CONCENTRÉ SUR LA DIGESTIBILITÉ ET LES QUANTITÉS INGÉRÉES DE RATIONS A BASE DE PANGOLA (DIGITARIA DECUMBENS) DISTRIBUE EN VERT A DES CHEVRES LAITIERES ET A DES BOUCS
}

\author{
M. CHENOST \\ avec la collaboration technique de P. Daury et P. Despois \\ Station de Recherches zootechniques, \\ Centre de Recherches agronomiques des Antilles et de la Guyane, I. N. R. A., \\ Petit-Bourg, Guadeloupe
}

\section{RÉSUMÉ}

Nous avons étudié l'influence de l'apport de quantités variables de deux aliments concentrés, l'un (A) à 2,9 p. Ioo, l'autre (B) à I I,3 p. Ioo (dont la moitié est apportée par de l'urée) d'azote total (tabl. I),

- d'une part sur les quantités de matière sèche ingérées par des chèvres en lactation et par des boucs consommant des rations à base de Pangola (Digitaria decumbens) d'âge et de digestibilité différents (tabl. I),

- d'autre part sur la digestibilité de la ration globale mesurée sur des boucs.

Les quantités de matière sèche totales ingérées ont augmenté en même temps que la quantité d'aliment concentré distribuée (fig. I, 3, 4).

Une augmentation de Io p. Ioo de la proportion de l'aliment concentré $\mathrm{A}$ dans la ration entraîne une diminution significative des quantités de matière sèche ingérées sous forme de fourrage d'autant moins rapide que la digestibilité de celui-ci est faible (I2o $\mathrm{g}$ et $2 \mathrm{I} 6 \mathrm{~g} / \mathrm{Ioo} \mathrm{kg}$ de poids vif respectivement pour des fourrages d'une digestibilité moyenne de 59,3 et 69,5 p. Ioo) (fig. 2). La digestibilité de la ration globale augmente en même temps que la proportion d'aliment concentré et cela d'autant plus que la digestibilité du fourrage distribué seul est faible (fig. 3).

Un apport de ${ } 50 \mathrm{~g}$ de concentré riche en azote (B) ne diminue pas les quantités de fourrage ingérées (fig. 4), et augmente de façon intéressante (5 à 6 points) la digestibilité globale de la ration (fig. 4). L'influence de la complémentation azotée sur la digestibilité des rations à base de fourrages tropicaux de faible digestibilité est donc importante.

\section{INTRODUCTION}

D'une manière générale, les graminées ont une valeur alimentaire plus faible en zone tropicale qu'en zone tempérée (MInson et MAC LEOD, I970) ; leur digestibilité et leur teneur en matières azotées digestibles sont en particulier moins élevées en zone tropicale; les quantités qui sont 
ingérées par le ruminant à qui on les présente à volonté sont également plus faibles. Pour assurer la couverture des besoins énergétique et azoté des animaux à fort potentiel de production, il est donc nécessaire que les graminées tropicales soient complémentées par une quantité importante d'aliments concentrés. La culture des graminées tropicales se développe cependant rapidement dans toute la zone des Caraïbes parce qu'elles ont une productivité et une longévité exceptionnelles ; c'est ainsi qu'une prairie de Pangola (Digitavia decumbens) exploitée intensivement peut produire 50 tonnes de matière sèche à l'hectare par an (SALETTE, 197o) et durer plus de vingt ans. En revanche, les variétés de légumineuses fourragères adaptées à cette zone tropicale humide sont moins productives et leur installation en prairie est encore délicate.

On sait que les quantités de fourrage ingérées, de foin et đ'ensilage notamment, diminuent lorsque la proportion d'aliment concentré dans la ration augmente, et cela d'autant plus que les fourrages ont une valeur nutritive plus élevée (Blaxter et WiLson, r963; CAMPLING et MurDoch, i966; Demarguilly, ig68, résultats non publiés; Chenost et Demarguilly, i969). On sait beaucoup moins ce qui se passe avec des fourrages verts notamment dans le cas de graminées tropicales.

Il était donc intéressant d'étudier l'influence de la quantité et de la teneur en azote de l'aliment concentré distribué aux animaux sur la digestibilité et la quantité ingérée des rations à base de Pangola. Cette étude a été effectuée sur des chèvres laitières à besoins élevés, et sur des boucs.

\section{MATÉRIEL, ET MÉTHODES}

\section{Fourrages}

Nous avons utilisé des repousses d'une prairie de Pangola (Digitaria decumbens) exploitée par la fauche, repousses âgées soit de 30 jours soit de 50 jours suivant les expériences. Pour cela la prairie avait été préalablement divisée en deux parties subđivisées l'une en 3o, l'autre en 50 parcelles, une parcelle correspondant à la surface fauchée chaque jour. La partie exploitée à $3^{\circ}$ jours d'âge recevait $840 \mathrm{~kg}$ d'azote et $840 \mathrm{~kg}$ de potasse/ha/an ; l'autre, exploitée à 50 jours d'âge, recevait $35^{\circ} \mathrm{kg}$ d'azote et $35^{\circ} \mathrm{kg}$ de potasse/ha/an; la fumure phosphorique a été respectivement égale à 250 et $\mathrm{I} 30 \mathrm{~kg}$.

Ces deux techniques d'exploitation nous ont permis d'obtenir des fourrages de qualité très différente (tabl. I).

\section{Aliment concentré}

Nous avons utilisé deux aliments concentrés, l'un (A) d'une teneur en azote de 2,9 p. roo, l'autre (B) d'une teneur en azote de I I,3 p. roo dont la moitié était apportée par de l'urée. La composition de ces deux aliments est donnée dans le tableau I.

\section{Animaux}

Les chèvres et les boucs étaient de race alpine (Saanen et Chamoisée), pesant respectivement

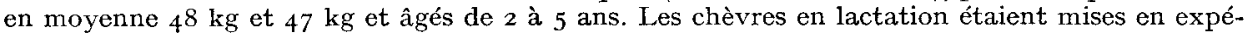
rience après le $2^{\mathrm{e}}$ mois de lactation.

\section{Dispositif expérimental}

\section{Essai 1 .}

Vingt-quatre chèvres en lactation ont été réparties en 6 lots de 4 animaux, 3 lots ont reçu les repousses de 30 jours, les 3 autres les repousses de 50 jours. L'herbe était fauchée une fois par jour le matin et distribuée individuellement en deux repas (début de matinée et fin d'après-midi). Chaque lot a été soumis à une succession de 3 niveaux de complémentation $\left(\mathrm{N}_{1}, \mathrm{~N}_{2}, \mathrm{~N}_{3}\right)$ avec l'aliment A suivant un protocole expérimental de type carré latin. La durée des périodes était de 5 semaines (3 préexpérimentales, 2 expérimentales). La complémentation était de $0,5 \mathrm{~kg}$ de concentré par $\mathrm{kg}$ de lait brut produit au-dessus de $\mathrm{I}, \mathrm{o} \mathrm{kg} / \mathrm{j}$ pour le niveau $\mathrm{N}_{1}$, au-dessus de o,5 kg/j pour le niveau $\mathrm{N}_{2}$ et au-dessus de o $\mathrm{kg} / \mathrm{j}$ pour le niveau $\mathrm{N}_{3^{*}}$ 


\section{TABLEAU I}

Composition des aliments concentrés et valeurs extrêmes et moyennes de la composition chimique et de la digestibilité des repousses de Pangola (Digitaria decumbens)

I. Aliments concentrés.

\begin{tabular}{|c|c|c|}
\hline \multirow[b]{2}{*}{ Constituants des aliments } & \multicolumn{2}{|c|}{ Nature de l'aliment } \\
\hline & $\underset{(\%)}{\operatorname{Aliment} A}$ & $\underset{(\%)}{\text { Aliment B }}$ \\
\hline Maïs ........... & 20 & - \\
\hline Orge $\ldots \ldots \ldots \ldots \ldots \ldots \ldots \ldots \ldots \ldots \ldots \ldots \ldots$ & 40 & - \\
\hline Tourteau de soja extraction ........... & - & 79 \\
\hline Tourteau de lin extraction $\ldots \ldots \ldots \ldots$ & 6 & 一 \\
\hline Tourteau d'arachide extraction...$\ldots \ldots$ & 18 & - \\
\hline Luzerne déshydratée $\ldots \ldots \ldots \ldots \ldots$ & 10 & - \\
\hline 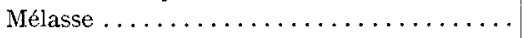 & - & 3 \\
\hline Urée $(47 \%$ N) $\ldots \ldots \ldots \ldots \ldots \ldots$ & - & 12 \\
\hline Condiment minéral vitaminé . . . . . . . & 6 & 6 \\
\hline Total .. & 100 & 100 \\
\hline $\mathrm{N}(\% \mathrm{MS}) \ldots$ & 2,88 & 11,35 \\
\hline
\end{tabular}

2. Repousses de Pangola.

\begin{tabular}{c|c|c}
\hline Nature du fourrage & $\begin{array}{c}\text { Repousses de 30 } \mathrm{j} \\
(\mathrm{N}=18)\end{array}$ & $\begin{array}{c}\text { Repousses de } 50 \mathrm{j} \\
(\mathrm{N}=18)\end{array}$ \\
\hline $\begin{array}{c}\text { Digestibilité } \\
\text { de la matière sèche (\%) }\end{array}$ & $\begin{array}{c}69,5 \\
(63,8-74,5)\end{array}$ \\
\hline $\begin{array}{c}\text { Matières azotées totales } \\
\text { (\% MS) }\end{array}$ & $\begin{array}{c}59,3 \\
(55,3-66,1)\end{array}$ \\
\hline $\begin{array}{c}\text { Cellulose brute de Weende } \\
(\% \text { MS) }\end{array}$ & $\begin{array}{c}32,1 \\
(6,5-12,8) \\
(32,2-35,9)\end{array}$ \\
\hline \hline
\end{tabular}

\section{Essai 2.}

Pendant l'expérience menée sur chèvres, 6 lots de 6 boucs (4 en cage à digestibilité, 2 en case au sol) ont reçu pendant 3 périodes différentes d'une durée de 2 I jours, les uns le fourrage de 3o jours, les autres le fourrage de 50 jours, additionné d'une quantité d'aliment A représentant, suivant les lots, 0,25 ou $50 \mathrm{p}$. Ioo de la matière sèche totale de la ration ; ces proportions ont été maintenues en corrigeant chaque jour le rationnement à partir des résultats de la mesure des quantités de matière sèche ingérées l'avant-veille. Cette modalité de distribution a été décidée à partir de l'étude des mesures des quantités ingérées par les chèvres ; elle avait surtout pour but de mesurer la digestibilité de rations comparables à celles ingérées par les chèvres en expérience. 


\section{Essai 3.}

Dans une troisième série d'expériences les boucs ont reçu, pendant 2 périodes de 2 I jours, le fourrage de 30 jours plus, suivant les lots, o, 50 ou Ioo g d'aliment $B$ riche en azote, puis pendant 3 périodes de $2 \mathrm{I}$ jours, le fourrage de 50 jours plus 0,50 , roo ou $\mathrm{I} 5 \mathrm{o}$ g de ce même aliment.

L'apport a été limité à Ioo et $5_{5} 0 \mathrm{~g}$ de façon à ne pas fournir aux boucs une quantité d'urée trop grande.

\section{Mesures}

Nous avons mesuré les quantités de matière verte et de matière sèche ingérées individuellement par les animaux par pesée des quantités distribuées et refusées et détermination de leur teneur en matière sèche. Les données de consommation correspondent pour les chèvres, à la moyenne des consommations journalières individuelles de 4 chèvres pendant 5 jours et pour les boucs, à la moyenne des consommations journalières individuelles de 6 boucs pendant io jours.

La digestibilité globale des rations (fourrages distribués soit seuls, soit avec l'aliment concentré) a été mesurée dans chaque essai avec 4 des 6 boucs de chaque lot.

Les teneurs en cendres, cellulose brute de Weende et matières azotées totales ont été déter. minées sur les échantillons représentatifs des fourrages proposés et refusés, des aliments concentrés et des fèces dans le cas des boucs.

\section{RÉSULTATS}

\section{Valeur nutritive des fourrages}

La composition moyenne et les limites de variation de la composition chimique et de la digestibilité des deux groupes de repousses de Pangola sont reportées dans le tableau r.

En moyenne, les repousses âgées de 30 jours ont eu une teneur en matières azotées (totales) supérieure $(\mathrm{P}<\mathrm{o}, \mathrm{OI})$ de 5,4 points (I2,I contre 6,7 p. IOo) et une teneur en cellulose brute inférieure de 2,7 points $(34,2$ contre 36,9 p. roo) à celles des repousses âgées de 50 jours. La digestibilité de la matière sèche des repousses âgées de 30 jours a été supérieure en moyenne de Io,2 points à celle des repousses âgées de 50 jours : 69,5 contre 59,3 p. Ioo. La valeur nutritive des deux groupes de fourrages verts utilisés a donc été très différente.

\section{Quantités ingérées: infuence de la quantité et de la nature de l'aliment concentré}

\section{Chèvres.}

Les quantités de matière sèche ingérées par roo $\mathrm{kg}$ de poids vif ont varié suivant les périodes de $\mathrm{I}, 68$ à $3,72 \mathrm{~kg}$; elles ont augmenté en même temps que la proportion d'aliment concentré $\mathrm{A}$ (fig. I). A proportion d'aliment concentré égale, elles ont été plus élevées avec les régimes à base de Pangola âgé de 3 o jours.

Les quantités d'aliment concentré ingérées ont représenté, suivant les périodes de mesure, de 90 à $\mathrm{I}$ ooo $\mathrm{g} / \mathrm{j}$, soit de 5 à $66 \mathrm{p}$. Ioo de la matière sèche de la ration totale avec les repousses de $5^{\circ}$ jours et de 90 à $85^{\circ} \mathrm{g} / \mathrm{j}$ soit de 3 à $56 \mathrm{p}$. Ioo de la matière sèche de la ration totale avec les repousses de $30 \mathrm{j}$.

Les quantités de matière sèche de fourrage ingérées ont toujours été plus élevées dans le cas des repousses de 30 jours que dans le cas des repousses de $5^{\circ}$ jours lorsque les fourrages étaient distribués seuls; elles ont diminué significativement $(\mathrm{P}<\mathrm{O}, \mathrm{OI})$ lorsque le rapport concentré/ total a augmenté (fig.2) et d'autant moins que le fourrage était de faible qualité quand il était distribué seul, ce qui est en accord avec les résultats de CAMPling et Murdoch (1966) sur foin et de Demarquilly (résultats non publiés) sur foin et ensilage. 
C'est ainsi que la quantité $(y)$ de matière sèche de fourrage ingérée par Ioo $\mathrm{kg}$ de poids vif est liée au pourcentage $(x)$ d'aliment concentré $\mathrm{A}$ dans la ration par les équations suivantes :

$$
\begin{aligned}
& y_{30 \mathrm{~J}}=2,77-0,02 \mathrm{I} 6 x \pm 0,256\left(n=\mathrm{I} 8 r=-0,840^{* *}\right) \\
& y_{50 \mathrm{~J}}=2,03-0,0120 x \pm 0,22 \mathrm{I}\left(n=\mathrm{I} 8 r=-0,763^{* *}\right)
\end{aligned}
$$

En moyenne l'ingestion de $100 \mathrm{~g}$ de matière sèche d'aliment concentré $\mathrm{A}$ a fait diminuer l'ingestion de Pangola âgé de 30 jours et 50 jours respectivement de $\mathrm{I}_{4} \mathrm{O}$ et $80 \mathrm{~g}$ de matière sèche.

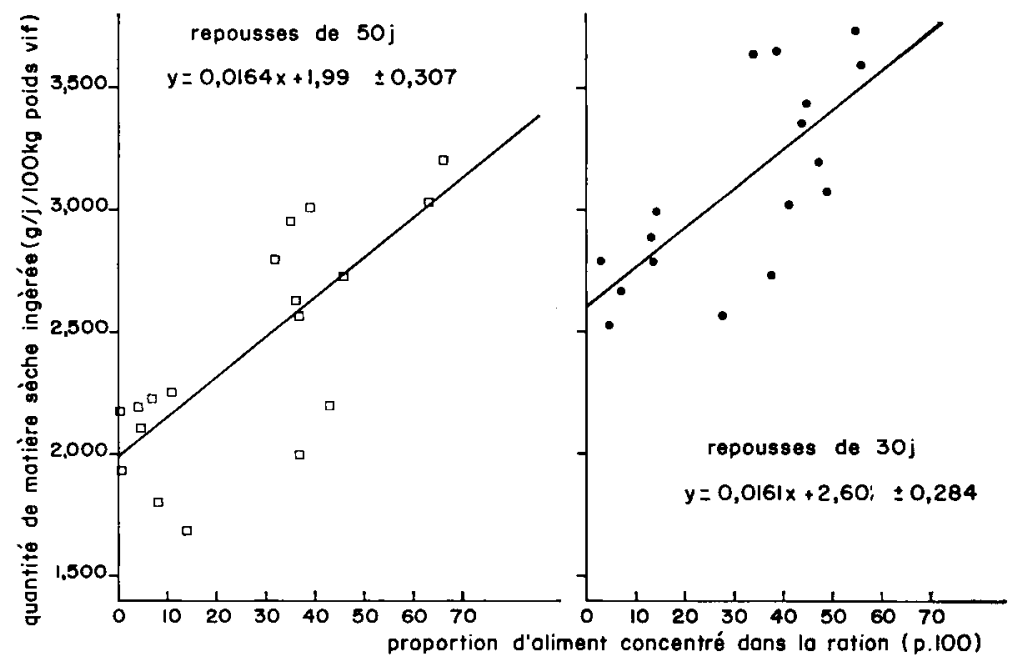

FIG. I. - Influence de la proportion d'aliment concentré $A$

sur les quantités totales de matière sèche ingérées par des chèvres laitières

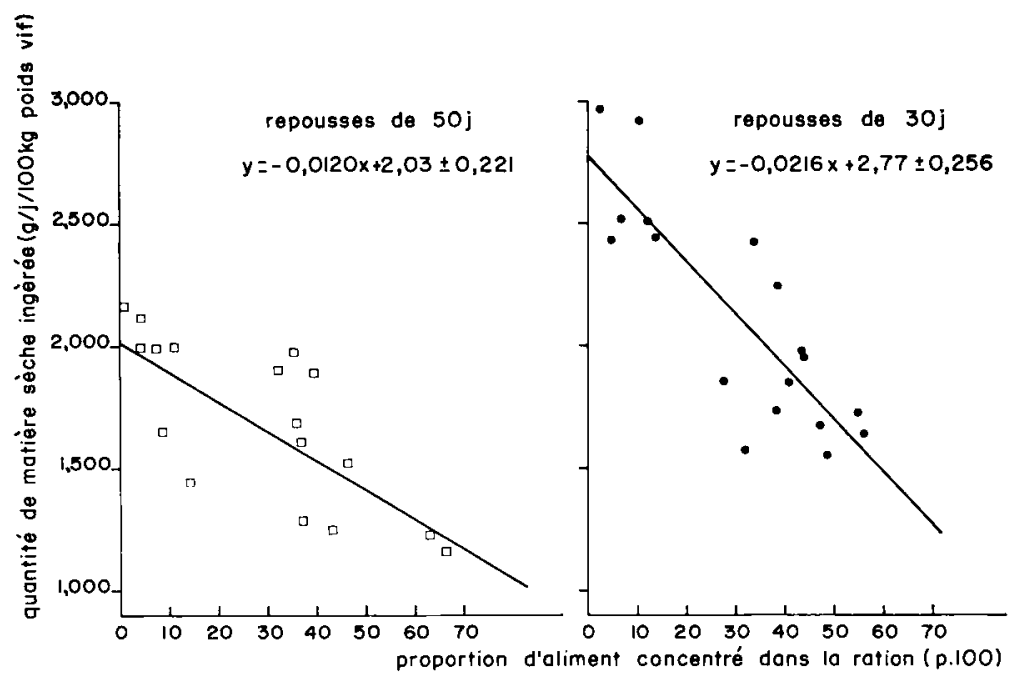

FIG. 2. - Influence de la proportion d'aliment concentré $A$

sur les quantités de matière sèche ingérées sous forme de fourrage par des chèvres laitières

\section{Boucs.}

Les quantités de matière sèche ingérées par roo $\mathrm{kg}$ de poids vif ont varié, suivant les essais, de I,I6 à 2,70 kg. Elles ont augmenté en même temps que la proportion d'aliment concentré et n'ont pas été différentes suivant l'âge du fourrage (fig. 3 et 4 ). 
Les quantités d'aliment concentré A ingérées ont représenté environ $250 \mathrm{~g}$ et $600 \mathrm{~g} / \mathrm{j}$ lorsqu'elles étaient distribuées, respectivement, à raison de 25 et $50 \mathrm{p}$. Ioo de la matière sèche totale ingérée. Les quantités d'aliment concentré $\mathrm{B}$ (inférieures ou égales à $15 \circ \mathrm{g} / \mathrm{j}$ ) n'ont jamais dépassé $20 \mathrm{p}$. roo de la ration totale.

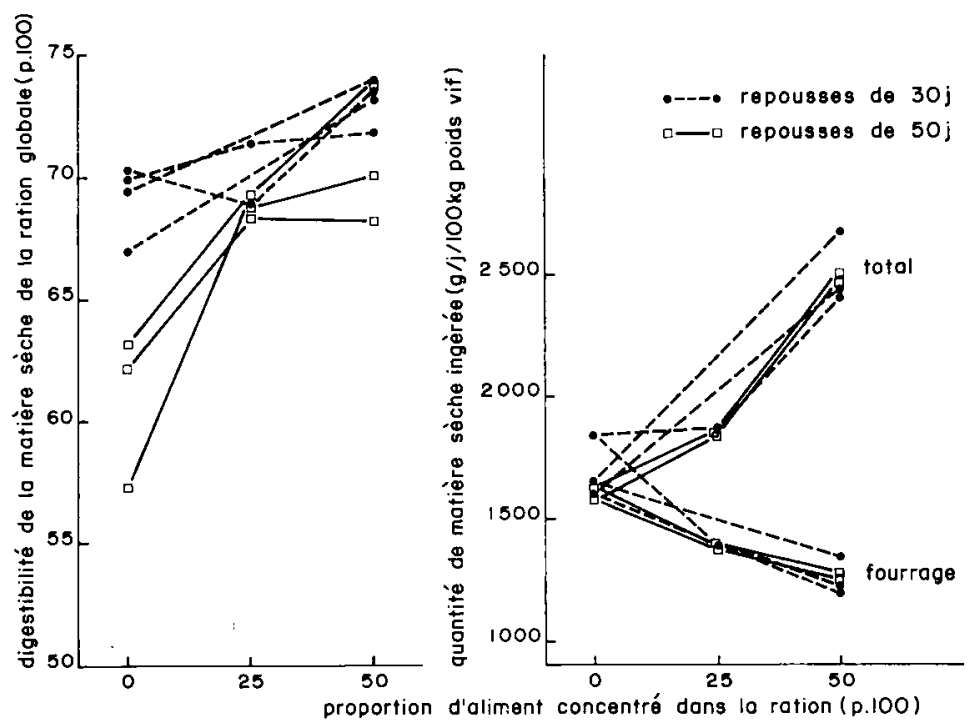

FIG. 3. - Influence de la proportion d'aliment concentré A dans la ration sur la digestibilité globale de la ration et sur les quantités de matière sèche totale et de fourrage

(Digitaria decumbens) ingérées par les boucs
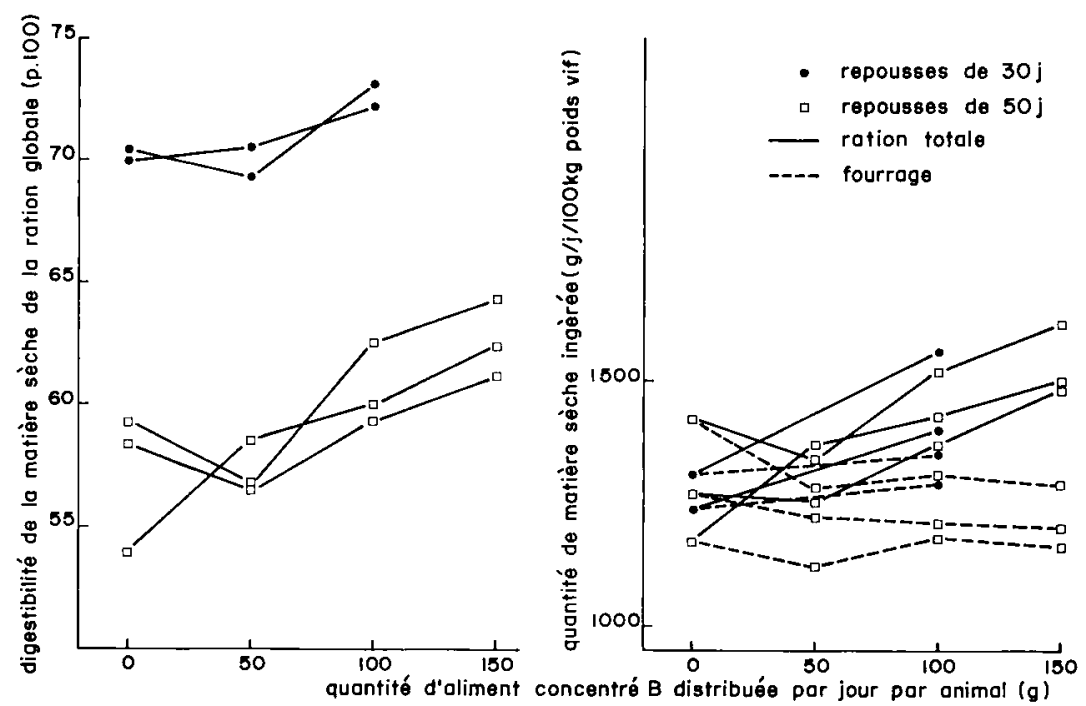

Fig. 4. - Influence de la quantité d'un aliment concentré riche en azote sur la digestibilité globale de la ration et sur les quantités de matière sèche totale et de fourrage

(Digitaria decumbens) ingérées par des boucs 
En moyenne l'ingestion de $100 \mathrm{~g}$ de matière sèche de l'aliment $\mathrm{A}$ a fait diminuer l'ingestion de Pangola de 80 à Ioo $\mathrm{g}$ de façon indépendante de l'âge des repousses. L'ingestion de l'aliment $B$ n'a pas modifié les quantités de fourrages ingérées (fig. 4).

\title{
Digestibilité de la ration totale
}

La digestibilité de la ration totale ingérée par les boucs a augmenté en même temps que la proportion d'aliment concentré dans la ration. Cette augmentation a été d'autant plus importante que la digestibilité du fourrage distribué seul était faible (fig. 3 et 4 ).

Il est intéressant de constater que même de faibles apports de l'aliment concentré riche en azote ont entraîné une augmentation importante ( 5 à 6 points) de la digestibilité (fig. 4). Celle-ci a toutefois été moins grande qu'avec l'aliment A qui était distribué en quantité beaucoup plus élevée. I1 semble donc que la complémentation en azote soit aussi déterminante de l'augmentation de la digestibilité globale des rations à base de fourrages tropicaux que la complémentation énergétique.

\section{DISCUSSION}

Ce travail doit être poursuivi sur un plus grand nombre de fourrages pour mesurer, d'une part, les quantités d'éléments nutritifs ingérés suivant la qualité des fourrages offerts, la nature et la quantité de l'aliment concentré distribué, d'autre part, l'utilisation de ces éléments nutritifs par des animaux en croissance, en engraissement et en lactation. Notre objectif est de déterminer, pour chaque catégorie d'animaux, suivant la qualité des fourrages qui leur seront offerts, la quantité et la nature (teneur et qualité de l'azote) de l'aliment concentré complémentaire nécessaire à la réalisation des performances zootechniques désirées tout en utilisant au maximum les fourrages qui peuvent être produits sur place en grande quantité.

Reçu pour publication en juillet 1971.

\section{SUMMARY}

\author{
INFLUENCE OF THE SUPPLY OF CONCENTRATES ON THE DIGESTIBILITY \\ AND VOLUNTARY INTAKE OF DIETS BASED ON PANGOLA GRASS \\ (DIGITARIA DECUMBENS), OFFERED AS GREEN FORAGE TO LACTATING \\ AND MALE GOATS.
}

Using lactating and male goats fed diets based on Pangola grass (Digitaria decumbens) of different ages and digestibilities (table $\mathrm{I}$ ), we studied the effect of supplying different amounts of two concentrates, A, containing 2.9 p. Ioo nitrogen and B, II.3 p. Ioo (half of it being constituted of urea) on the dry matter intake, and on the digestibility of the whole ration measured on male goats only.

The total dry matter intake was increased when the supplements were offered (fig. I,3 and 4).

When increasing, by to per cent, the supply of concentrate $A$ in the diet, there was a significant reduction of the dry matter intake in the form of forage, which was all the more slow as the digestibility of the latter was low ( $\mathrm{I} 20 \mathrm{~g}$ and $2 \mathrm{I} 6 \mathrm{~g} / \mathrm{I} 00 \mathrm{~kg}$ live weight respectively for forages with a mean digestibility of 59.3 and 69.5 per cent) fig. 2 . The digestibility of the whole ration increased at the same time as the proportion of concentrate, increase that was all the more important as the digestibility of the forage allotted alone was low (fig. 3). 
A supply of $50 \mathrm{~g}$ of the rich nitrogen concentrate (B) did not reduce the forage intake and improved digestibility of the total diet by 5-6 points (fig. 4). Thus, the nitrogen supplementation of diets based on tropical forages of low digestibility has an important effect on the digestibility of these diets.

\section{RÉFÉRENCES BIBLIOGRAPHIQUES}

Blaxter K. L., Wirson R. S., I963. The assessment of a crop husbandry technique in terms of animal production. Anim. Prod., 5, 27-42.

CAmpling R. C., Murdoch J. C., I966. The effect of concentrates on the voluntary intake of roughages by cows. J. Dairy Res., 38, I-II.

Chenost M., Demarquilly C., r969. Comparaison entre le pâturage et l'affouragement en vert pour la production de viande bovine. Ann. Zootech., 18, $277-298$.

Minson D. J., MaC Leod M. N., I97o. The digestibility of temperate and tropical grasses. Proc. XIth inter. grassl. Congr., 719-722.

Salette J. E., 1970. Nitrogen use and intensive management of grass in the wet tropics. Proc. XIth inter. Grassl. Cong., 404-407. 